\title{
Perforación y obstrucción colónica: conducta de los coloproctólogos latinoamericanos
}

\author{
Günther Bocic A. ${ }^{1}$, Mauricio Zambra R. ${ }^{1}$, Mario Abedrapo M. ${ }^{1}$, Antonella Sanguinetti M. ${ }^{1}$, \\ José L. Llanos B. ${ }^{1}$, Rodrigo Azolas M. ${ }^{1}$, Mauricio Díaz B. ${ }^{1}$, Felipe Ignacio Bocic W. ${ }^{2}$, \\ Tomas Rodríguez C. ${ }^{1}$ y Natalia Moreno B. ${ }^{1}$
}

'Departamento de Cirugía Hospital Clínico Universidad de Chile. Santiago, Chile. ${ }^{2}$ CESFAM Federico Puga Borne. Servicio de Salud Ñuble. Ñuble, Chile.

Recibido 2019-12-16 y aceptado 2020-02-15

Correspondencia a: Dr. Günther Bocic A guntherbocic@hotmail.com
Perforation and obstruction of the colon: behavior of the latin american colon and rectal surgeons

Objective: Learn about the different management options performed by latin american colon and rectal surgeons, in relation to colonic emergencies. Materials and Method: Cross-sectional study, using web survey with multiple-choice questions. The survey was sent to the different Latin America Coloproctological Societies, as well as to ALACP, for distribution. The survey was written in neutral Spanish and translated into Portuguese. Descriptive and analytical statistical analysis was used. Results: 441 complete surveys, from 16 different countries. $85 \%$ perform resection and anastomosis without diverting ostomy in obstructions of the right colon. In perforations of the left colon, Hartmann's procedure is performed in $63.3 \%$ of case with purulent peritonitis and in $94.5 \%$ of fecaloid peritonitis. Discussion: In colonic obstructions, resection with primary anastomosis, is little discussed behavior in the right colon, unlike obstructions on the left side, where performing a Hartmann operation is a behavior as valid as resection and anastomosis. In colonic perforation, the decision of resection and primary anastomosis is multifactorial, taking into account the hemodynamic stability of the patient. In the latter cases, performing an ostomy is the response of a large part of the surveyed. Conclusions: The results in each situation, for the most part, present a clear tendency towards a particular behavior; only in the case of left colon obstruction, two behaviors (Hartmann procedure or primary anastomosis) are both validated by international literature.

Keywords: low intestinal obstructions; purulent peritonitis; fecaloid peritonitis; Hartmann procedure.

\section{Resumen}

Objetivo: Conocer las diferentes conductas que realizan los cirujanos coloproctólogos latinoamericanos en relación con las urgencias colónicas. Materiales y Método: Estudio transversal, utilizando encuesta vía web con preguntas de selección múltiple. La encuesta fue enviada a las distintas Sociedades Coloproctológicas Latinoamericanas, así como a la Asociación Latinoamericana de Coloproctología (ALACP), para su distribución. La encuesta fue escrita en español neutro y traducida al portugués. Se utilizó análisis estadísticos descriptivos y analítico. Resultados: 441 encuestas respondidas completamente de 16 países diferentes. El $85 \%$ realiza resección y anastomosis sin ostomía de protección en obstrucciones de colon derecho. En las perforaciones del colon izquierdo, se realiza operación de Hartmann en el 63,3\% de los casos que presentan peritonitis purulentas y en el 94,5\% de las peritonitis fecaloideas. Discusión: En las obstrucciones colónicas, la resección con anastomosis primaria, es una conducta poco discutida en colon derecho, a diferencia de las obstrucciones del lado izquierdo, en donde realizar una operación de Hartmann es una conducta tan válida como la resección y anastomosis. En los cuadros de perforación, la decisión de resección y anastomosis primaria es multifactorial, tomando relevancia la estabilidad hemodinámica del paciente. En estos últimos casos, realizar una resección con ostomía, es la respuesta de gran parte de los encuestados. Conclusiones: Los resultados de cada situación, en su mayoría, presentan una tendencia clara hacia una conducta en particular; solo en el caso de obstrucción de colon izquierdo, se observan dos conductas (operación de Hartmann o anastomosis primaria) ambas validadas por la literatura internacional. Palabras clave: obstrucción intestinal baja; peritonitis purulenta; peritonitis fecaloídea; operación de Hartmann. 


\section{Introducción}

La patología colónica de urgencia cada vez tiene mayor prevalencia. Tomando en consideración todas las emergencias gastrointestinales, cerca del $50 \%$ corresponden a complicaciones de alguna patología colónica, se destacan dentro de estas complicaciones: la obstrucción intestinal y la perforación no traumática. Las etiologías más frecuentes son el cáncer colorrectal (CCR) y la diverticulitis aguda. El 30\% de los CCR se presentan con síntomas que requieren intervención quirúrgica de urgencia, de estos el $80 \%$ por obstrucción intestinal y el $20 \%$ por perforación ${ }^{1,2}$. El enfrentamiento a dichas urgencias colónicas son un real desafío para el cirujano general como para el coloproctólogo. En una primera instancia, es vital la reanimación con fluídos y los antibióticos para, posteriormente, definir la cirugía a realizar ${ }^{3}$. El espectro de tratamientos quirúgicos es amplio, debiendo tomar en cuenta la hemodinamia del paciente, la etiología, el pronóstico de la enfermedad y la experiencia del cirujano ${ }^{2-4}$. En relación a lo último, se ha demostrado en diferentes artículos, que la participación de un coloproctólogo es de vital importancia para definir el tipo de cirugía a realizar, asociado a que realizan menos ostomías y presentan menos tasas de filtración de anastomosis ${ }^{2,5,6}$.

Aun cuando no existen estudios prospectivos randomizados y grupos homogéneos, siempre al realizar el tratamiento quirúrgico se debe preferir la resección del segmento afectado y la anastomosis primaria $^{2,7}$.

Esta premisa debe ser evaluada y considerada según cada contexto en particular dependiente de un conjunto de factores externos, prefiriendo en algunas ocasiones realizar otras técnicas quirúrgicas que, por muy sencillas y poco resolutivas que parezcan, salvan la vida del paciente y mejoran su pronóstico a largo plazo ${ }^{2}$.

Actualmente, en la literatura latinoamericana de alto impacto, no existen consensos publicados sobre el manejo quirúrgico de las patologías anorrectales benignas, urgencias colónicas y cáncer colorrectal, por lo cual nos planteamos como objetivo, sondear y conocer las conductas quirúrgicas frente a diferentes situaciones en urgencias colónicas, que realizan los cirujanos coloproctólogos de Latinoamérica. Teniendo esto en mente y asumiendo que Chile será sede del Congreso de la Asociación Latinoamericana de Coloproctología (ALACP) en el año 2023, el objetivo final es, permitirnos durante el congreso, sentar las bases para el desarrollo de consensos latinoamericanos.

\section{Materiales y Método}

Se trata de un estudio tipo transversal, utilizando una encuesta vía web con preguntas de selección múltiple, la cual fue aplicada y respondida entre diciembre de 2017 y noviembre de 2018. La encuesta fue enviada a las distintas Sociedades Coloproctológicas Latinoamericanas, así como a ALACP, para su distribución en los coloproctólogos. La encuesta fue escrita en español neutro y traducida al portugués. Se incluyeron en el analisis solo las encuestas completadas en un 100\%. Se utilizó análisis estadísticos descriptivos y analítico, utilizando test de Chi-cuadrado.

\section{Resultados}

Se analizaron 441 encuestas $100 \%$ completas, de 16 países diferentes (Figura 1), la edad promedio de los coloproctólogos fue de 53 años con un rango entre 32-82 años. Cuando analizamos las respuestas en relación a obstrucción de colon, encontramos que el $85 \%$ realiza resección y anastomosis sin ostomía de protección en obstrucciones de colon derecho (Figura 2). En las obstrucciones de colon izquierdo, el 35\% realiza anastomosis sin ostomía de protección y el 45\% operación de Hartmann, sin existir diferencia significativa entre estas dos respuestas (Figuras 3 y 4).

En relación a la conducta que se toma frente a la perforación colónica derecha con peritonitis purulenta o fecaloidea, encontramos que, en la primera, el 47,2\% realiza anastomosis sin ostomía de protección, a diferencia de cuando existe peritonitis fecaloidea, en donde el 70,2\% realiza resección con ostomía proximal y distal. Cuando hablamos de perforación en colon izquierdo la mayoría prefiere realizar operación de Hartmann, un $63,3 \%$ en caso de peritonitis purulenta y un $94,5 \%$ en peritonitis fecaloidea (Tabla 1).

\section{Discusión}

Los resultados principales de este estudio suguieren que:

a) La mayoría de los encuestados refiere realizar resección y anastomosis sin ostomía de protección, frente al cuadro clínico de obstrucción intestinal baja de colón derecho.

b) Frente a un cuadro clínico de obstruccion intestinal baja con etiología en colon izquierdo, no hay una tendencia clara hacia una conducta específi- 
Figura 1. Distribución de coloproctólogos que respondieron la encuesta.

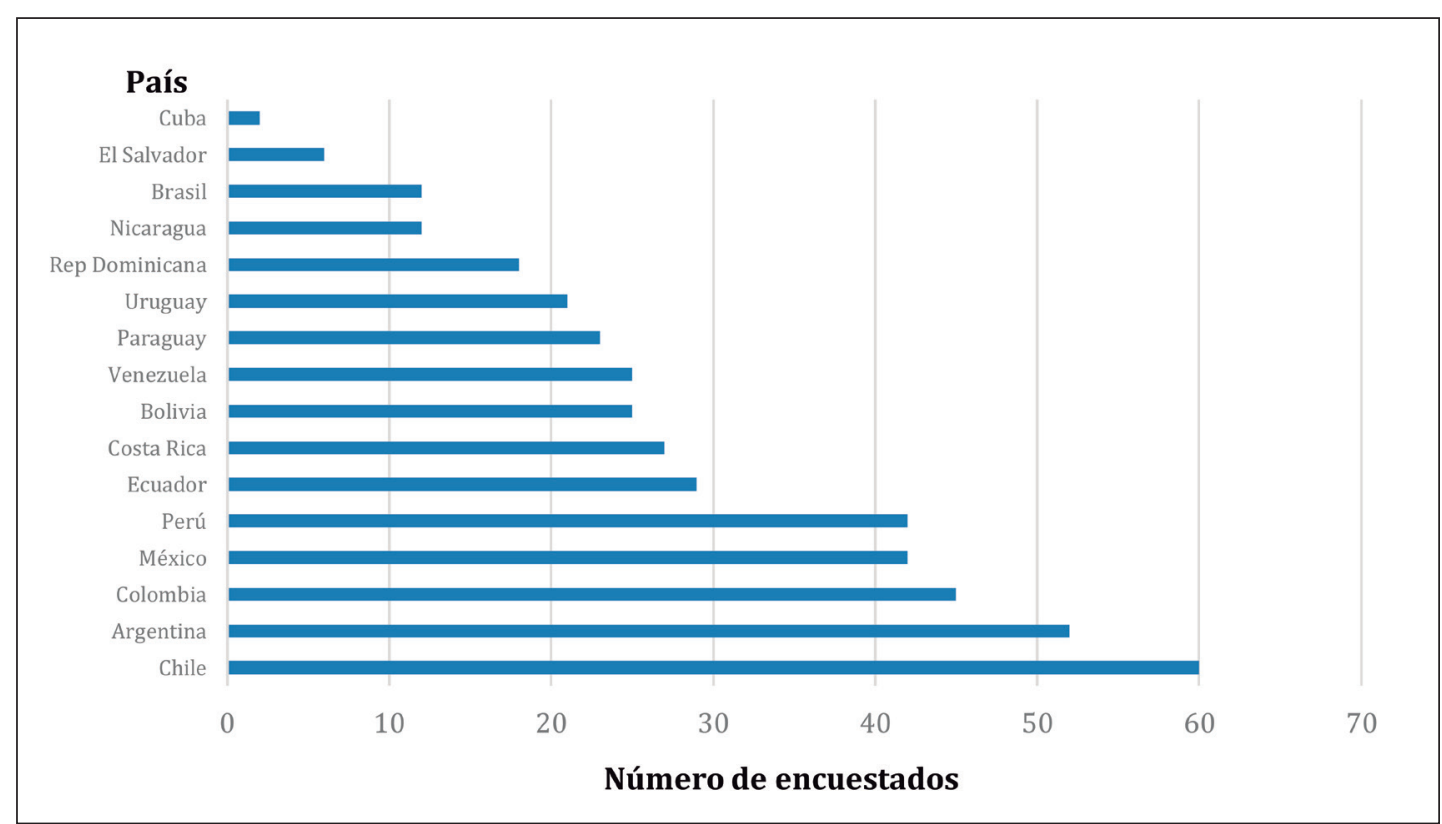

- Resección y anastomosis sin ostomía de protección

- Resección y ostomía proximal más distal

- Resección y anastomosis con ostomía de protección

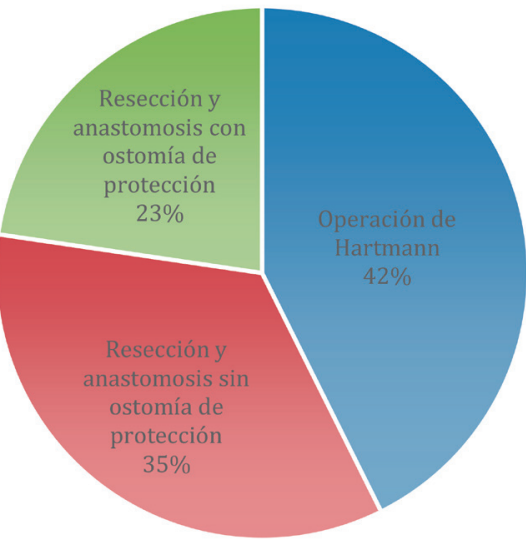

Figura 3. En caso de obstrucción de colon izquierdo, ¿cuál técnica utiliza con mayor frecuencia? $(n=441)$.

ca, algunos prefieren realizar una operación de Hartmann y otros, una resección con anastomosis sin ostomía de protección.

c) En perforaciones de colon derecho con peritonitis purulenta, la mayoría menciona que realiza una resección y anastomosis sin ostomía de protección.

d) Realizar una resección sin anastomosis, fue la respuesta de la mayoría, cuando se enfrentan a cuadros clínicos compatibles con: peritonitis fecaloidea de colon derecho, peritonitis purulenta de colon izquierdo y peritonitis fecaloidea de colon izquierdo. 
La obstrucción intestinal baja, sigue siendo una patología frecuente a resolver en la cirugía abdominal, siendo un diagnóstico de ingreso frecuente a los servicios de urgencia y cirugía ${ }^{8}$. La principal causa es el $\mathrm{CCR}^{2}$. La obstrucción es la indicación más común de cirugía de urgencia en el CCR, llegando a ser un $77 \%$ según algunas series ${ }^{9}$. La cirugía a realizar en este contexto debe tener como objetivos lo siguiente: 1) evitar el impacto negativo inmediato de la complicación; 2) lograr el mejor control oncológico; 3) asegurar una recuperación oportuna para el inicio apropiado de algún tratamiento sistémico ${ }^{7}$.

En general, cuando existe una obstrucción del colon derecho, la decisión sobre qué cirugía a realizar es poco discutida, esta debe ser la hemicolectomía derecha y anastomosis ileocolónica, la cual presenta un bajo riesgo de complicación. En nuestro trabajo esta fue la respuesta del $85 \%$ de los encuestados. Si bien se pueden asociar ileostomías de protección o derivativas, estas se deben realizar en pacientes graves o cuando la lesión que produce la obstrucción es imposible de resecar por su tamaño y/o adherencias a otros órganos o planos anatómicos ${ }^{10-12}$.

La obstrucción de colon izquierdo es más frecuente y, comparado con las derechas, existen más alternativas quirúrgicas que se deben discutir y ajustar para cada caso en particular ${ }^{2,4}$. En la actualidad hay dos tendencias claras, la operación de Hartmann y la resección con anastomosis primaria no protegida $^{2,7}$. En nuestra comunicación observamos que estas dos opciones fueron las más contestadas, sin existir diferencia significativa entre ambas. Durante muchos años la operación de Hartmann, ha sido las más realizada, con índices de mortalidad cercanos al $20 \%$ y de morbilidad de $30 \%$. Esta técnica elimina el riesgo de una dehiscencia, pero deja al paciente con una ostomía, requiriendo un segundo tiempo quirúrgico.

Diversos artículos hacen ver que al menos en un 40\%-50\% de los casos no se vuelve a reconstituir el tránsito intestinal ${ }^{3,13-15}$, incluso hay series que muestran que un $90 \%$ de los pacientes con operación de Hartmann de urgencia, por causa neoplásica, no se reconstituyen ${ }^{2}$, por la necesidad de adyuvancia o recidiva tumoral. La resección con anastomosis primaria sin ostomía de protección, es una cirugía segura; si bien no se han realizado estudios prospectivos comparativos entre Hartmann y anastomosis primaria, los porcentajes de complicación en esta última son comparables con la cirugía electiva, reportandose porcentajes de filtración de $2,2 \%-12 \%$ en cirugías de urgencia y de $2 \%-8 \%$ en la cirugía electiva ${ }^{2,7}$.

Cuando nos enfrentamos a una perforación colónica, lo que va a orientar nuestro tratamiento medi-
Tabla 1. Técnicas utilizadas según cuadro clínico

n $(\%)$

Perforación de colon derecho y peritonitis purulenta $(n=441)$

Resección y anastomosis sin ostomía de protección

$208(47,2)$

Resección y ostomía proximal+distal

$147(33,3)$

Resección y anastomosis con ostomía de protección

Perforación de colon derecho y peritonitis fecaloidea $(n=441)$

Resección y ostomía proximal+distal

$310(70,2)$

Resección y anastomosis con ostomía de protección

Resección y anastomosis sin ostomía de protección

Perforación de colon izquierdo y peritonitis purulenta $(n=441)$

Operación de Hartmann

$279(63,3)$

Resección y anastomosis con ostomía de protección

Resección y anastomosis sin ostomía de protección

Perforación de colon izquierdo y peritonitis fecaolidea $(n=441)$

Operación de Hartmann

Resección y anastomosis con ostomía de protección

$22(5,0)$

Resección y anastomosis sin ostomía de protección

co-quirurgico, va a ser la condición hemodinámica del paciente. En este caso, la clínica más frecuente es resistencia muscular, ausencia de ruidos hidroaéreos y signos de irritación peritoneal. La presencia de leucocitosis con desviación izquierda y/o acidosis láctica definen un cuadro séptico grave y pronóstico desfavorable $\mathrm{e}^{10,16-18}$

Es importante tener en cuenta que casi todos los estudios demuestran que la magnitud y calidad de la peritonitis no es un factor independiente que influya en la mortalidad operatoria y, por lo tanto, no debería ser una determinante significativa a considerar por sí sola al momento de decidir la técnica quirúrgica a realizar ${ }^{1,10,19,20}$. Tanto en perforaciones de colon derecho e izquierdo la recomendación de resección con anastomosis primaria, es una decisión supeditada al compromiso séptico del paciente más que al tipo de peritonitis ${ }^{21}$.

En nuestro estudio, el $47 \%$ de los coloproctólogos realizarían una anastomosis primaria sin ostomía de protección en el caso de perforación con peritonitis purulenta del colon derecho, si bien la pregunta no menciona otros factores, como el compromiso hemodinámico del paciente, posiblemente los especialistas toman esta conducta al saber que la anastomosis ileocolónica tiene tasas de filtración más bajas que las colo-colónicas o colorrectales. Se destaca que $33 \%$, en el mismo caso, prefirió realizar una resección sin anastomosis, la cual es una conducta aceptada y bien respaldada. 
Ante un paciente inestable se recomienda la resección con ileostomía terminal y fístula mucosa en lado derecho o Hartmann al lado izquierdo ${ }^{21}$. Estas conductas fueron las respuestas con mayor porcentajes en el resto de las situaciones clínicas, debido posiblemente a lo grave que significa una peritonitis fecaloidea y al mayor riesgo de filtración de anastomosis que existe en el colon izquierdo.

Si bien este reporte refleja, posiblemente, la realidad o la conducta de mayor prevalencia entre los cirujanos coloproctólogos de Latinoamérica, no podemos dejar de mencionar un sesgo importante, que es lo cerrada de las preguntas, las cuales no mencionan otros factores relevantes, como el compromiso hemodinámico, las que son de vital importancia al momento de tomar la decisión quirúrgica. Si bien es cierto, obtuvimos un buen número de respuestas, estas podrían haber sido más minuciosas y haber entregado información más completa.

\section{Conclusión}

Las respuestas de la encuesta aplicada, nos muestran conductas quirúrgicas comparables con la literatura actual de países desarrollados. Los resultados de cada situación, en su mayoría, presentan una tendencia clara hacia una conducta en particular; solo en el caso de obstrucción de colon izquierdo, se observan dos conductas (operación de Hartmann o anastomosis primaria) ambas validadas por la literatura internacional. Existe consenso en realizar ostomías en casos de peritonitis, tanto fecaloideas como purulentas.

Durante las últimas décadas, en las urgencias colónicas, el uso de colostomía derivativa era prácticamente de regla, sin embargo, con el paso de los años el péndulo se ha movilizado y se preconizan actualmente las resecciones de buena calidad, oncológicas y con anastomosis primaria cuando corresponda. Dichas cirugías deben ser realizadas por cirujanos con experiencia, pues sus resultados son similares, en cuanto a morbimortalidad, comparado con cirugías electivas.

A pesar de los sesgos, este trabajo sienta las bases para iniciar el desarrollo de un consenso en el año 2023, cuando se desarrolle el Congreso Latinoamericano de Coloproctología en Chile.

\section{Responsabilidades éticas}

Protección de personas y animales. Los autores declaran que para esta investigación no se han realizado experimentos en seres humanos ni en animales.

Confidencialidad de los datos. Los autores declaran que en este artículo no aparecen datos de pacientes.

Conflictos de interés: no hay.

\section{Bibliografía}

1. Zielinski MD, Merchea A, Heller SF, You YN. Emergency Management of Perforated Colon Cancers: How Aggressive Should We Be? J Gastrointest Surg. 2011;15:2232-8.

2. Pisano M, Zorcolo L, Merli C, Cimbanassi S, Poiasina E, Ceresoli M, et al. 2017 WSES guidelines on colon and rectal cancer emergencies: obstruction and perforation. World J Emerg Surg. 2018;13:36. doi: 10.1186/s13017-0180192-3. eCollection 2018.

3. Brown CV. Small bowel and colon perforation. Surg Clin North Am. 2014;94:471-5. doi:10.1016/j. suc.2014.01.010.

4. Sperry J, Cohen MJ. Acute obstruction. Surg Clin North Am. 2014;94:77-96. doi:10.1016/j.suc.2013.10.001

5. Tekkis PP, Kinsman R, Thompson
MR, Stamatakis JD; Association of Coloproctology of Great Britain, Ireland. The Association of Coloproctology of Great Britain and Ireland study of large bowel obstruction caused by colorectal cancer. Ann Surg. 2004;240:76-81. doi: 10.1097/01. sla.0000130723.81866.75.

6. Darby CR, Berry AR, Mortensen N. Management variability in surgery for colorectal emergencies. Br J Surg. 1992;79:206-10. doi:10.1002/ bjs. 1800790305 .

7. Baer C, Menon R, Bastawrous S, Bastawrous A. Emergency Presentations of Colorectal Cancer. Surg Clin North Am. 2017;97:529-45. doi:10.1016/j. suc. 2017.01.004.

8. Markogiannakis H, Messaris E, Dardamanis D, Pararas N, Tzertzemelis D, Giannopoulos P, et al. Acute mechanical bowel obstruction: clinical presentation, etiology, management and outcome. World J Gastroenterol. 2007;13:432-7. doi:10.3748/wjg.v13. i3.432.

9. Teixeira F, Akaishi EH, Ushinohama AZ, Dutra TC, Couto Netto SD, Utiyama EM, et al. Can we respect the principles of oncologic resection in an emergency surgery to treat colon cancer? World J Emerg Surg 2015 10, 5 https://doi. org/10.1186/1749-7922-10-5.

10. Gunnarsson H, Holm T, Ekholm A, Olsson LI. Emergency presentation of colon cancer is most frequent during summer. Colorectal Dis. 2011;13:663-8.

11. Shimura T, Joh T. Evidence-based Clinical Management of Acute Malignant Colorectal Obstruction. J Clin Gastroenterol. 2016;50:273-85. doi:10.1097/MCG.0000000000000475.

12. Hsu TC. Comparison of one-stage resection and anastomosis of acute 
complete obstruction of left and right colon. Am J Surg. 2005;189:384-7.

13. Trompetas V. Emergency management of malignant acute left-sided colonic obstruction. Ann R Coll Surg Engl. 2008;90:181-6. doi: 10.1308/003588408X285757.

14. Meyer F, Marusch F, Koch A, Meyer L, Fuhrer S, Kockerling F, et al. German Study Group "Colorectal. Emergency operation in carcinomas of the left colon: value of Hartmann's procedure. Tech Coloproctol. 2004;(8 Suppl 1):s226-9.

15. Alves A, Panis Y, Mathieu P, Mantion G, Kwiatkowski F, Slim K. Association Francaise de Postoperative mortality and morbidity in French patients undergoing colorectal surgery: results of a prospective multicenter study. Arch
Surg. 2005;140:278-83. doi: 10.1001/ archsurg.140.3.278.

16. Kim J, Mittal R, Konyalian V, King J, Stamos MJ, Kumar RR. Outcome analysis of patients undergoing colorectal resection for emergent and elective indications. Am Surg. 2007;73:991-3.

17. Bellows CF, Webber LS, Albo D, Awad $\mathrm{S}$, Berger DH. Early predictors of anastomotic leaks after colectomy. Tech Coloproctol. 2009;13:41-7. doi: 10.1007/ s10151-009-0457-7.

18. Yang XF, Pan K. Diagnosis and management of acute complications in patients with colon cancer: bleeding, obstruction, and perforation. Chin J Cancer Res. 2014;26:331-40. doi: 10.3978/j.issn.1000-9604.2014.06.11

19. Biondo S, Kreisler E, Millan M,
Fraccalvieri D. Differences in patient postoperative and long-term outcomes between obstructive and perforated colonic cancer. Am J Surg. 2008;195:42732. doi: 10.1016/j.amjsurg.2007.02.027.

20. Anwar MA, D'Souza F, Coulter R, Memon B, Khan IM, Memon MA. Outcome of acutely perforated colorectal cancers: experience of a single district general hospital. Surg Oncol. 2006;15:916. doi: 10.1016/j.suronc.2006.09.001.

21. Veyrie N, Ata T, Muscari F, Couchard AC, Msika S, Hay JM, et al. French Associations for Surgical Research Anastomotic leakage after elective right versus left colectomy for cancer: prevalence and independent risk factors. J Am Coll Surg. 2007;205:785-93. doi: 10.1016/j.jamcollsurg.2007.06.284 